\title{
Antagonism of p66shc by melanoma inhibitory activity
}

\author{
K Kasuno $^{1}$, A Naqvi ${ }^{1}$, J DeRicco ${ }^{1}$, T Yamamori ${ }^{1}$, L Santhanam ${ }^{2}$, I Mattagajasingh ${ }^{1}$, S Yang ${ }^{3}$, FL Meyskens ${ }^{3}$, A-K Bosserhoff ${ }^{4}$ and \\ K Irani ${ }^{*, 1}$
}

The p66shc protein governs oxidant stress and mammalian lifespan. Here, we identify melanoma inhibitory activity (MIA), a protein secreted by melanoma cells, as a novel binding partner and antagonist of p66shc. The N-terminal collagen homology-2 (CH2) domain of p66shc binds to the Src Homology-3 (SH3)-like domain of MIA in vitro. In cells, ectopically expressed MIA and p66shc colocalize and co-precipitate. MIA also co-precipitates with the $\mathrm{CH} 2$ domain of p66shc in vivo. MIA expression in vivo suppresses p66shc-stimulated increase in endogenous hydrogen peroxide $\left(\mathrm{H}_{2} \mathrm{O}_{2}\right)$, and inhibits basal and $\mathrm{H}_{2} \mathrm{O}_{2}$-induced phosphorylation of p66shc on serine 36 and $\mathrm{H}_{2} \mathrm{O}_{2}$-induced death. In human melanoma cells expressing MIA, endogenous MIA and p66shc co-precipitate. Downregulation of MIA in melanoma cells increases basal and ultraviolet radiation (UVR)-induced phosphorylation of p66shc on serine 36 , augments endogenous $\mathrm{H}_{2} \mathrm{O}_{2}$ levels, and increases their susceptibility to UVR-induced death. These findings show that MIA binds to p66shc, and suggest that this interaction antagonizes phosphorylation and function of p66shc.

Cell Death and Differentiation (2007) 14, 1414-1421; doi:10.1038/sj.cdd.4402131; published online 13 April 2007

P66shc belongs to the shcA family of adapter proteins, and plays a crucial part in governing mammalian lifespan, the only protein thus far shown to do so. In this regard, p66shc-null mice live 30\% longer than their wildtype littermates, and are resistant to oxidant stimuli. ${ }^{1}$ In response to such stimuli, p66shc is phosphorylated on serine 36 , a residue that is not present in other shcA proteins. ${ }^{2}$ The phosphorylation of p66shc on serine 36 is essential to its function as a governor of cellular reactive oxygen species levels. ${ }^{3}$

P66shc governs reactive oxygen species levels by regulating mitochondrial oxidative capacity. ${ }^{4}$ Recently, we have reported another mechanism through which p66shc increases oxidant stress: by stimulating the activity of the guanine nucleotide exchange factor son of sevenless- 1 toward the rac1 GTPase. ${ }^{5}$ This mechanism employs interaction between specific proline residues in the unique $\mathrm{N}$-terminal collagen homology-2 ( $\mathrm{CH} 2)$ domain of p66shc to the src homology-3 (SH3) domains of growth factor receptor bound-2 (Grb2). The ability of the proline-rich $\mathrm{CH} 2$ domain to bind to $\mathrm{SH} 3$ domains of grb2 raised the possibility of other $\mathrm{SH} 3$-containing proteins as binding partners of p66shc.

Melanoma Inhibitory Activity (MIA) is a protein that plays a key role in the progression and metastasis of malignant melanoma. Its name, a misnomer, is derived from its initial characterization as a protein secreted by a human melanoma cell line that inhibited attachment and growth of melanoma cells in culture. ${ }^{6}$ Subsequently, MIA was shown to bind to specific extracellular matrix proteins, thereby masking the binding sites of integrins to these proteins, ${ }^{7}$ as well as directly binding to specific integrins. ${ }^{8}$ MIA promotes melanoma metastasis and invasion in vivo, ${ }^{9}$ partly through these means. MIA is primarily expressed in malignant melanoma cells, though emerging evidence indicates that other cell types, especially chondrocytes, and particularly other tumor cells, also express varying amounts of MIA. ${ }^{10-15}$ In humans, serum MIA is useful in detecting progression of localized melanoma to metastatic disease, ${ }^{16}$ and also for monitoring therapy of advanced melanomas. ${ }^{17}$ MIA belongs to a select group of small proteins that adopt a single $\mathrm{SH} 3$ domain-like structure, ${ }^{7}$ and was identified as the first secreted protein with a SH3-like domain.

To identify novel binding partners of p66shc, we screened an array of $\mathrm{SH} 3$ domains using the $\mathrm{CH} 2$ domain of p66shc as bait. We found that the SH3-like domain of MIA bound avidly to the $\mathrm{CH} 2$ domain in vitro. Furthermore, we show that MIA is a bona fide binding partner of p66shc in vivo, characterize the molecular interaction that mediates binding between MIA and p66shc, and demonstrate the impact of this binding on the known physiologic functions of p66shc.

\section{Results}

P66shc contains a 110 amino-acid stretch at its $\mathrm{N}$-terminus that is not present in other shcA proteins. This $\mathrm{N}$-terminal region, termed the collagen homology-2 domain, is rich in proline and leucine residues, and is a potential binding partner

\footnotetext{
${ }^{1}$ Cardiovascular Institute, University of Pittsburgh Medical Center, Pittsburgh, PA, USA; ${ }^{2}$ The Johns Hopkins University, Baltimore, MD, USA; ${ }^{3}$ University of CaliforniaIrvine, Orange, CA, USA and ${ }^{4}$ University of Regensburg Medical School, Regensburg, Germany

${ }^{*}$ Corresponding author: K Irani, Cardiovascular Institute, University of Pittsburgh Medical Center, 623S Scaife Hall, 200 Lothrop St., Pittsburgh, PA, 15213, USA.

Tel.: + 412648 9229; Fax: + 412648 5991; E-mail: iranik@upmc.edu

Keywords: Melanoma; p66shc; oxidative stress

Abbreviations: Shc, Src homologous and collagen-like; MIA, melanoma inhibitory activity; $\mathrm{CH}$, collagen homology; Sos, son of sevenless; grb, growth factor receptor bound; ROS, reactive oxygen species; MEF, mouse embryonic fibroblasts; WT, wild type; UVR, ultraviolet radiation; JNK, c-jun N-terminal kinase; ERK, extracellular signal-regulated kinase; OTOR, otoraplin; His, histidine; SDS-PAGE, sodium dodecyl sulfate polyacrylamide gel electrophoresis; EGFP, enhanced green fluorescent protein; GTP, guanine triphosphate; GDP, guanine diphosphate; OD, optical density; ELISA, enzyme-linked immunsorbent assay; Bcl-2, B-cell chronic lymphocyte leukemia-2; ER, endoplasmic reticulum; BiP, binding protein; GST, glutathione S-transferase

Received 03.10.06; revised 07.2.07; accepted 28.2.07; Edited by RA Knight; published online 13.4.07
} 
of proteins with $\mathrm{SH} 3$ domains. ${ }^{5}$ To identify novel binding partners of p66shc, recombinant $\mathrm{CH} 2$ was used as bait in an in vitro screening assay. The SH3-like domain of MIA was identified as a strong binding partner of the $\mathrm{CH} 2$ domain by far-Western blotting an array of $\mathrm{SH} 3$ domains (Figure 1).

Armed with this finding we asked if MIA binds to p66shc in vivo. Ectopic MIA and p66shc expressed in COS7 cells colocalized (Figure 2a) primarily in the peri-nuclear region (Figure 2a). To determine if endogenous MIA and p66shc associate with each other, we utilized a human melanoma cell line HMB2 that expresses MIA, and its derivative cell lines, HMB2-5 in which endogenous MIA expression is suppressed with stable expression of a MIA anti-sense construct, and HMB2-LacZ that stably expresses a control LacZ construct. ${ }^{18}$ Fractionation of homogenates from MIA-expressing HMB2 cells demonstrated that MIA was principally present in the endoplasmic reticulum, which also had the majority portion of p66shc (Figure 2b). MIA also co-precipitated with p66shc and the isolated $\mathrm{CH} 2$ domain of p66shc, when expressed in COS7 cells (Figures $2 \mathrm{c}-\mathrm{e}$ ). Furthermore, immunoprecipitation of endogenous MIA in HMB2 and HMB2-LacZ cells co-precipitated p66shc, and lack of MIA in HMB2-5 cells led to no precipitation of p66shc (Figure $3 a$ and b). Similar to HMB2-5 cells, in HEK 293 cells that do not express MIA, MIA antibody did not pull down endogenous p66shc (Figure 3a).

Specific proline residues in the $\mathrm{CH} 2$ domain mediate its binding to the $\mathrm{SH} 3$ domain(s) of the growth factor receptor bound protein grb2. ${ }^{5}$ Since MIA adopts a SH3 domain-like structure, we assessed whether these proline residues are important in binding of p66shc to MIA. Compared to wild-type p66shc, binding of p66shc mutated at these proline residues (P47A/P50A) was significantly diminished (Figure 2d).

We then wondered if this association between MIA and p66shc has any functional significance. P66shc mediates the generation of the ROS $\mathrm{H}_{2} \mathrm{O}_{2}$, and cell death induced by oxidative stimuli. ${ }^{2}$ We therefore asked if MIA could modulate p66shc-mediated $\mathrm{H}_{2} \mathrm{O}_{2}$ generation and cell death. In COS7 cells, overexpression of p66shc enhanced $\mathrm{H}_{2} \mathrm{O}_{2}$-stimulated cell death that was rescued by co-expression of MIA (Figure 4a). Similarly, expression of p66shc in p66shc -/-

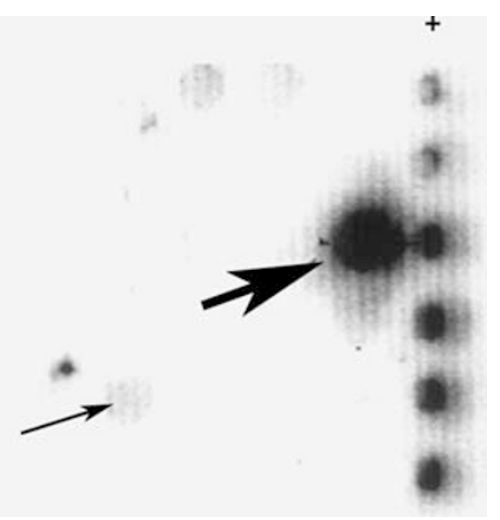

Figure $1 \mathrm{MIA}$ binds to the $\mathrm{CH} 2$ domain of p66shc in vitro. Far-Western blot of a spotted array of $\mathrm{SH} 3$ domains (SH3 domain array III, www.panomics.com) probed with the recombinant $\mathrm{CH} 2$ domain of p66shc. Bold arrow indicates position of spotted MIA, and thin arrow the position of the MIA homolog OTOR. + indicates column of positive control spots
MEF promoted $\mathrm{H}_{2} \mathrm{O}_{2}$-stimulated death that was rescued by coexpression of MIA (Figure $4 \mathrm{~b}$ and $\mathrm{c}$ ). In contrast to wild-type p66shc, expression of the non-phosphorylatable p66shcS36A mutant in p66shc -/- MEF did not increase $\mathrm{H}_{2} \mathrm{O}_{2}$-stimulated cell death (Figures $4 \mathrm{~b}$ and $\mathrm{c}$ ). Furthermore, MIA did not suppress $\mathrm{H}_{2} \mathrm{O}_{2}$-induced death in p66shc - /- cells expressing p66shcS36A (Figures 4b and c). In parallel with these findings, expression of p66shc in p66shc - /- MEF led to an increase in endogenous $\mathrm{H}_{2} \mathrm{O}_{2}$, and co-expression of MIA inhibited this increase (Figure 4d). In contrast, in p66shc -/- MEF in which exogenous p66shc was not expressed, MIA did not have any effect on basal endogenous $\mathrm{H}_{2} \mathrm{O}_{2}$, whereas in p66shc $+/+$ MEF (WT-MEF), MIA expression markedly reduced endogenous $\mathrm{H}_{2} \mathrm{O}_{2}$ levels (Figure 4e). These findings show that MIA suppresses $\mathrm{H}_{2} \mathrm{O}_{2}$ levels only in cells that express p66shc, and the capacity of MIA to suppress p66shc-stimulated $\mathrm{H}_{2} \mathrm{O}_{2}$, and inhibit oxidative stress-induced cell death, is only apparent in the context of phosphorylatable p66shc-MIA has no effect on $\mathrm{H}_{2} \mathrm{O}_{2}$ levels and cell viability in the context of phosphorylationdeficient, functionally inactive, p66shc.

Since our previous work had suggested that one mechanism by which p66shc increase $\mathrm{H}_{2} \mathrm{O}_{2}$ is by activating rac $1,{ }^{5}$ we examined whether MIA expression has any impact on rac1 activity. In WT-MEF expressing endogenous rac1, MIA did not suppress active rac1 levels (Figure 4f).

To determine the role of endogenous MIA in regulating cell survival and ROS levels, we compared $\mathrm{H}_{2} \mathrm{O}_{2}$ levels and susceptibility to oxidative stress-mediated death in $\mathrm{HMB} 2$, HMB2-LacZ and HMB2-5 cells. Basal, steady-state endogenous $\mathrm{H}_{2} \mathrm{O}_{2}$ levels were significantly higher in the MIA-deficient HMB2-5 cells when compared to HMB2 and HMB2-LacZ cells (Figure $5 \mathrm{a}$ ). In addition, $\mathrm{H}_{2} \mathrm{O}_{2}$-induced cell death was markedly greater in HMB2-5 cells when compared to the other two cell lines (Figures $5 \mathrm{~b}$ and $\mathrm{c}$ ). Forced reconstitution of MIA in MIA-deficient HMB2-5 cells led to a partial rescue against $\mathrm{H}_{2} \mathrm{O}_{2}$-induced cell death (Figure $5 \mathrm{~d}$ ). Ultraviolet radiation (UVR), another stimulus that leads to cell death through oxidative stress, also resulted in a much higher proportion of death in HMB2-5 cells, as compared to the MIA-expressing HMB-2 and HMB-LacZ cells (Figure 5e).

Next, we looked into a possible mechanism by which binding of MIA to p66shc may inhibit the function of the latter. Guided by the finding that MIA associates with the $\mathrm{CH} 2$ domain, and that MIA has no effect on death in cells expressing the phosphorylation-deficient p66shcS36A mutant, we hypothesized that the association of MIA and wild-type p66shc inhibits the phosphorylation of serine 36 in the $\mathrm{CH} 2$ domain, which is essential for the pro-oxidative and pro-apoptotic functions of p66shc. ${ }^{1,3,19}$ To test this hypothesis we first examined basal and $\mathrm{H}_{2} \mathrm{O}_{2}$-stimulated phosphorylation of serine 36 in COS7 cells. Expression of MIA suppressed both basal and $\mathrm{H}_{2} \mathrm{O}_{2}$-stimulated phosphorylation of serine 36 in endogenous and ectopic p66shc (Figure $6 a$ and b). Since serine 36 is a target of c-jun N-terminal kinases (JNKs), ${ }^{20}$ we wondered if MIA suppresses phosphorylation of serine 36 by inhibiting JNK activity. Basal, $\mathrm{H}_{2} \mathrm{O}_{2}$ and UVR-induced phosphorylated (active) JNKs was not different between MIAexpressing and non-expressing cells (Figure $6 c$ and $d$ ), suggesting that MIA-induced decrease in phosphorylation of serine 36 is not due to a decrease in JNK activity. We also 
a
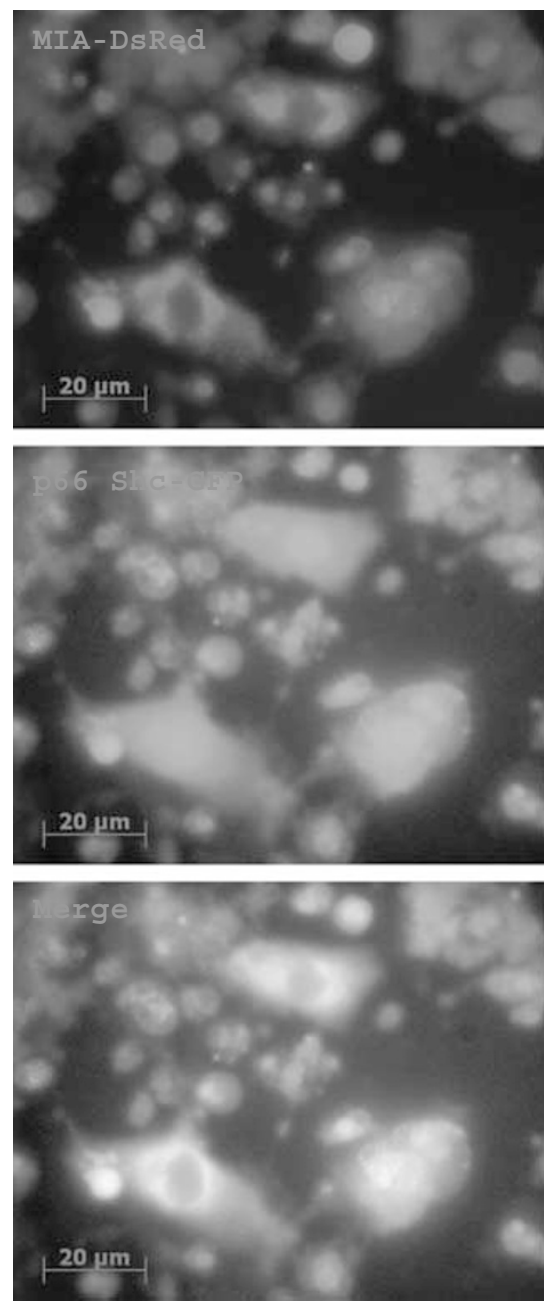

b



C



d
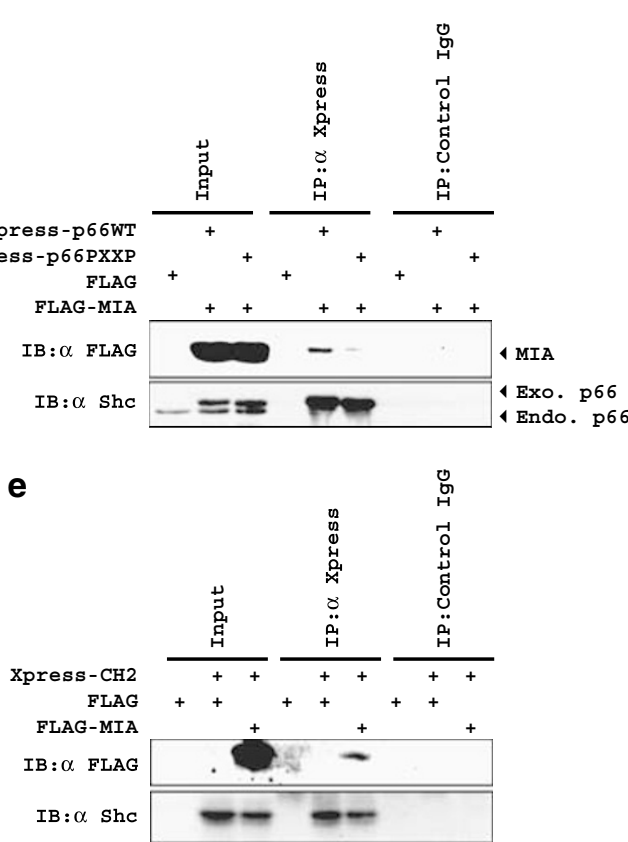

Figure 2 P66shc associates with MIA in vivo via proline residue in its $\mathrm{CH} 2$ domain. (a) Colocalization of MIA and p66 Shc in COS-7 cells. Live red, and green fluorescence images of COS-7 cells co-transfected with MIA-DsRed and pEGFP-p66shc were captured and merged. Nuclei were counterstained with Hoechst 33342 . (Original magnification $\times 400$ ). (b) Co-compartmentalization of MIA and p66shc. Immunoblots of fractionated lysates of MIA-expressing HMB2 melanoma cells. Identity of the fraction was verified by immunoblotting for proteins exclusively expressed in those fractions. Nucleus: $\mathrm{p} 300$ histone acetylase; Mitochondria: B-cell chronic lymphocyte leukemia-2 (Bcl-2); Endoplasmic Reticulum (ER): Binding Protein/GRP78 (BiP). (c) Co-immunoprecipitation of ectopically expressed FLAG-MIA and endogenous p66shc in COS-7 cells. (d) Co-immunoprecipitation of ectopically expressed FLAG-MIA and Xpress-p66shcWT or the mutant p66shcP47A/P50A in COS-7 cells. (e) Co-immunoprecipitation of ectopically expressed FLAG-MIA and Xpress-CH2 (the $\mathrm{CH} 2$ domain of p66shc) in COS-7 cells

looked at phosphorylation of serine 36 in the HMB2 and HMB2-5 melanoma cell lines. Basal and UVR-induced phosphorylation of p66shc on serine 36 was higher in the MIA-deficient HMB2-5 cells when compared to MIA-expressing HMB2 and HMB2-LacZ cells (Figure 7a and b). Moreover, this higher degree of basal p66shc phosphorylation in HMB2-5 cells was abrogated by forced reconstitution of MIA (Figure 7c). In addition to the HMB2 human melanoma cell line, MIA expression in a murine B16 melanoma-derived cell line was also associated with less phosphorylation of endogenous p66shc on S36, and lower endogenous $\mathrm{H}_{2} \mathrm{O}_{2}$ levels, when compared to a B16 melanoma cell line that does not express MIA (Figure 7d).

\section{Discussion}

Malignant melanoma has high metastatic potential, responds poorly to radiation and chemotherapy, and metastatic 
a

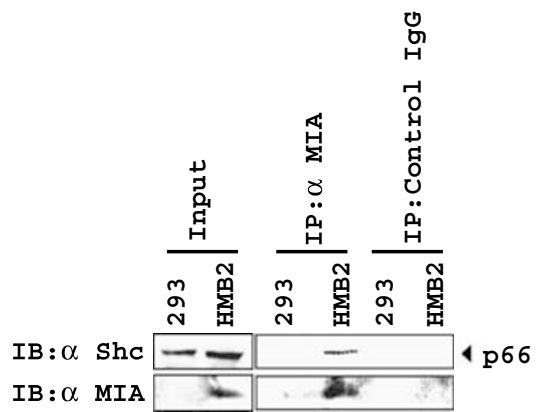

b

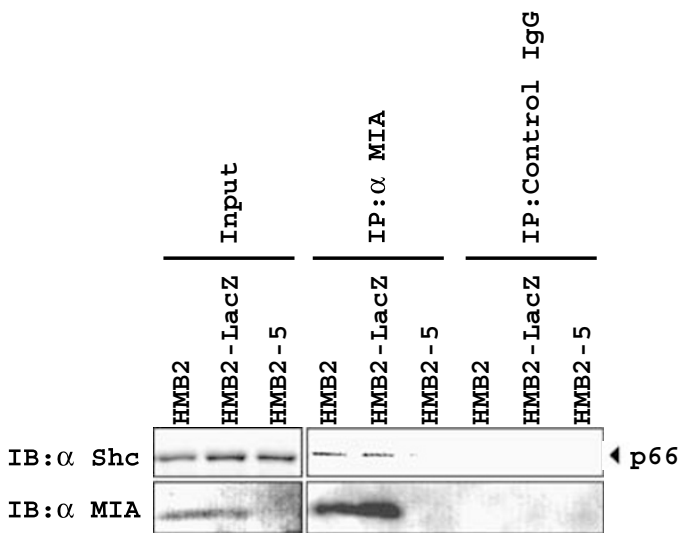

Figure 3 Endogenous MIA and p66shc associate in human melanoma cells. (a) Co-immunoprecipitation of endogenous MIA and p66Shc in the human metastatic melanoma cell line HMB2. Lysate from HEK 293 cells that do not express MIA was used as a control. (b) Co-immunoprecipitation of endogenous MIA and p66Shc in HMB2 cells, and its derivative cell lines HMB2-LacZ (HMB2 cells stably transfected with the inert $E$. coli LacZ gene), and HMB2-5 (HMB2 cells stably transfected with the antisense CDNA for MIA)

melanoma cells are less susceptible to apoptosis in vivo than non-metastatic cells. ${ }^{21}$ Moreover, MIA expression correlates with progression of melanocytic tumors, and predicts response of melanoma to traditional therapeutic modalities. ${ }^{17,22}$ Our findings, by identifying MIA as a novel binding partner of p66shc, and demonstrating that this binding inhibits p66shc-mediated $\mathrm{H}_{2} \mathrm{O}_{2}$ generation, suggest that one mechanism by which MIA may confer a survival advantage to melanoma cells expressing it, is by inhibiting p66shcmediated oxidative stress and cell death induced by irradiation and traditional chemotherapeutic agents. As such, strategies aimed at disrupting MIA-p66shc interaction may offer novel therapies to combat malignant metastatic melanoma.

The functional role of p66shc in cancer cells, much less melanoma cells, is controversial. In prostate cancer cell lines expression of p66shc correlates with proliferation, ${ }^{23}$ whereas in other tumor cell lines p66shc plays an apoptotic role. ${ }^{19}$ Although we did not directly examine the role of p66shc in proliferative capacity of melanoma cells, comparison of UVR- and $\mathrm{H}_{2} \mathrm{O}_{2}$-induced death in MIA-expressing and nonexpressing cells suggests that in this specific context (in MIA-expressing melanoma cells subjected to these oxidative cytotoxic stimuli) p66shc functions in a pro-apoptotic fashion. In this regard, it should be noted that although initially described as a protein restricted to melanoma cells, it is now appreciated that various malignant tumors express MIA. ${ }^{10,11,13}$ Therefore it is tempting to speculate that the antagonistic functional relationship between MIA and p66shc may not be relevant exclusively to melanoma cells.

MIA is secreted by melanoma cells, and its extracellular function as an inhibitor of integrin-mediated cell adhesion has been described. ${ }^{7,24}$ MIA has also been reported to localize in the intracellular space ${ }^{25}$ but its function as an intracellular protein is not known. It is known that melanoma cells expressing MIA display alterations in specific intracellular signaling pathways, such as activation of extracellular signalregulated kinases (ERKs). ${ }^{18}$ The mechanism by which MIA leads to activation of the ERK cascade is unclear. However, it is interesting to note that p66shc blunts ERK activation. ${ }^{19}$ This reciprocal relationship between p66shc and MIA with regard to ERK activity lends further support to our conclusion that MIA acts as a functional antagonist to p66shc.

MIA is the first member of a family of structurally alike small proteins that assume an SH3-like fold. Other members of this family include OTOR whose expression is limited to the inner ear, ${ }^{26}$ MIA2 that is principally expressed in the liver, ${ }^{27}$ and TANGO which is expressed in most tissue except that of hematopoetic origin. ${ }^{28}$ Although the $\mathrm{N}$-terminal regions of MIA, OTOR, MIA2, and TANGO are quite divergent, many residues important for structural folding are conserved between the four proteins. Our in vitro screening assay did detect an interaction between the $\mathrm{CH} 2$ domain and OTOR (Figure 1), albeit weaker than between the $\mathrm{CH} 2$ domain and MIA. This array does not have MIA2 and TANGO, and therefore we could not screen for an interaction between these two proteins and the $\mathrm{CH} 2$ domain. Nevertheless, the possibility that p66shc may bind to structurally conserved MIA homologs that are more broadly expressed than MIA suggests that the physical and functional interaction between MIA and p66shc that we describe in melanoma cells, may have wider implications in regulating oxidative stress, and cell death in other tissues and cells types.

It is important to recognize that MIA may impact only on select mechanisms by which p66shc functions. For example, p66shc-induced oxidative stress is partly mediated by rac $1,{ }^{5}$ but MIA expression does not suppress rac1 activity (Figure 4f). This selectivity likely reflects the compartmentalization of MIA within the cell. MIA, which is expressed in the endoplasmic reticulum, would not be expected to interfere with the binding of p66shc to Grb2 (an interaction that is required for p66shc-induced rac1 activation), a protein primarily present at the plasma membrane.

Collectively, these findings identify MIA as a novel and direct binding partner of p66shc, and show that this interaction has a functional consequence vis-à-vis the role of p66shc in promoting oxidative stress and cell death. This data also suggests that p66shc through interaction with MIA, which is expressed in a number of malignancies other than melanoma, may counteract the role of MIA in promoting tumor growth and metastases.

\section{Materials and Methods}

Cell lines, cDNA, and transfections. The metastatic human melanoma cell line and its derivatives, the murine B16 melanoma cell line and its derivatives, and the cDNA for human MIA, have been described previously. ${ }^{9,18}$ The cDNA for 

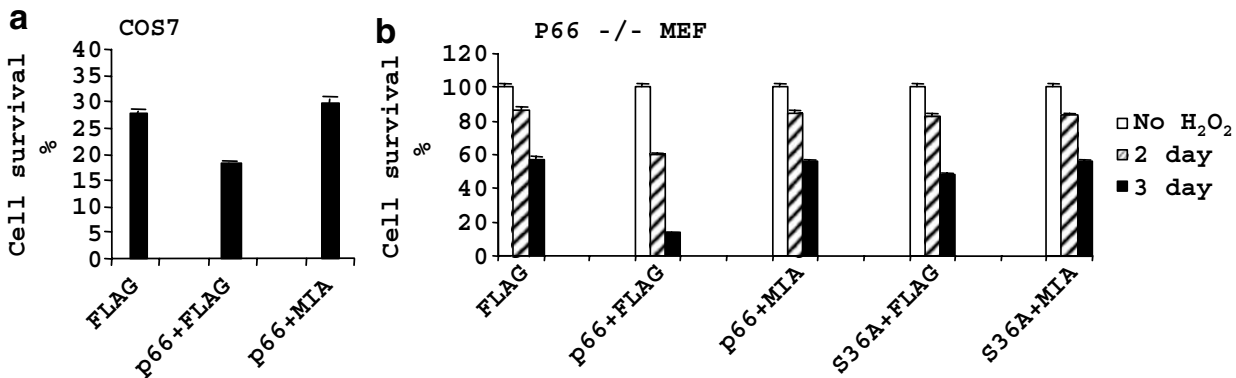

C

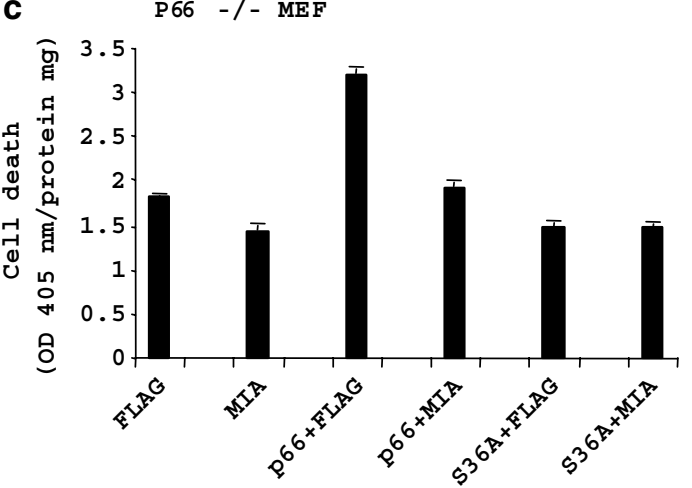

d
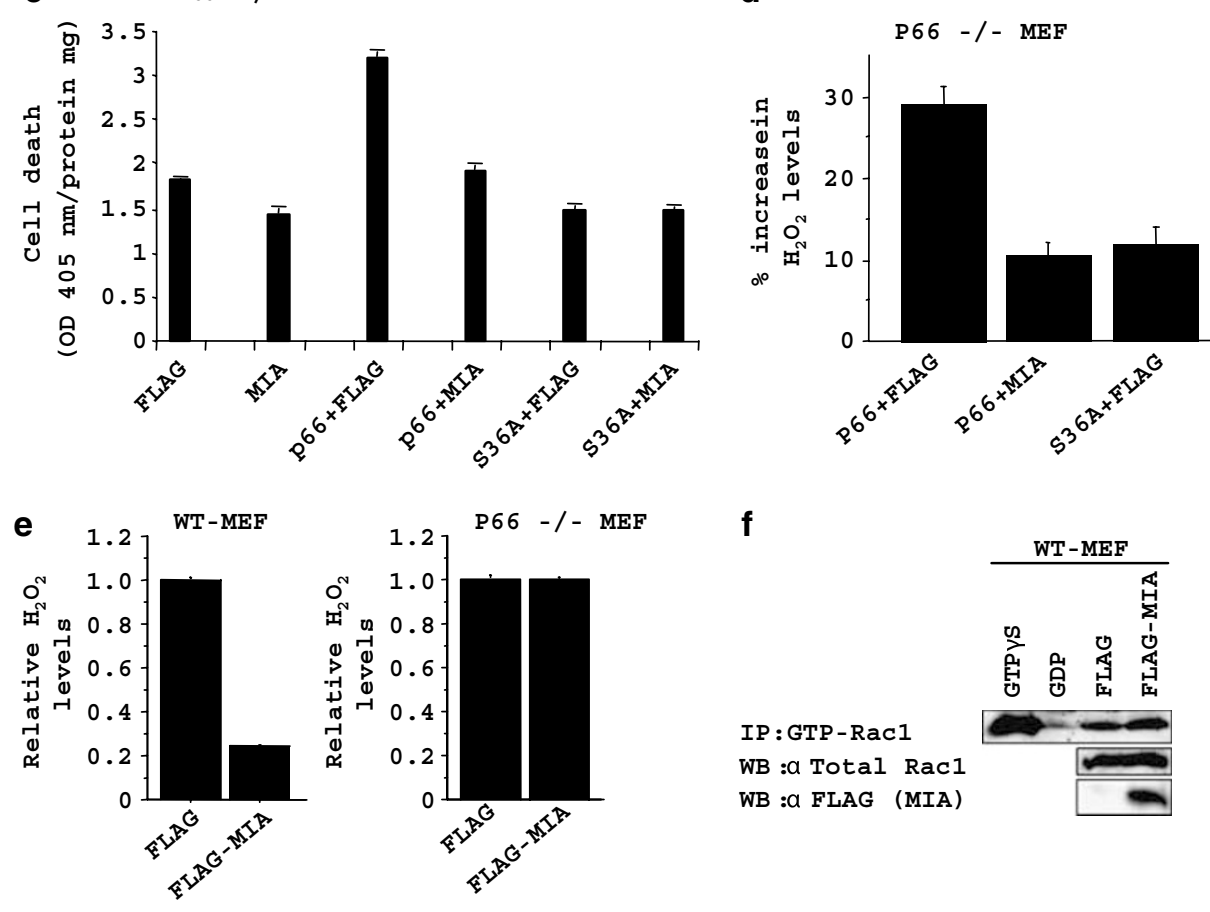

f


Figure 4 MIA suppresses p66shc-mediated, oxidative stress-induced cell death. (a) Cell survival, measured by Trypan blue exclusion, in COS-7 cells overexpressing p66shc with and without MIA. Cells were challenged with $0.4 \mathrm{mM} \mathrm{H}_{2} \mathrm{O}_{2}$ for $48 \mathrm{~h} . \mathrm{N}=4$, mean \pm S.E. Importance of serine 36 (S36) in the $\mathrm{CH} 2$ domain of p66shc to protection conferred by MIA. Cell survival at 24 and $48 \mathrm{~h}$ by Trypan blue exclusion (b) and cell death measured at $48 \mathrm{~h}$ by ELISA (c) in p66shc-null mouse embryonic fibroblasts (p66shc $-/-\mathrm{MEF}$ ) after challenged with $0.4 \mathrm{mM} \mathrm{H}_{2} \mathrm{O}_{2} \cdot \mathrm{N}=4$, mean \pm S.E. Cells were transfected with p66shcWT (p66) or the S36A mutant of p66shc (S36A), with or without MIA. (d) MIA inhibits p66shc-induced endogenous $\mathrm{H}_{2} \mathrm{O}_{2}$ levels. Endogenous hydrogen peroxide levels were measured by Amplex Red fluorescence in p66shc $-I-\mathrm{MEF}$ transfected with p66shcWT (p66) or p66shcS36A (S36A), with and without MIA. $N=7$, mean \pm S.E. (e) Effect of MIA expression on $\mathrm{H}_{2} \mathrm{O}_{2}$ levels in p66shc $+I+($ WT-MEF), and p66shc $-I-$ MEF. Endogenous $\mathrm{H}_{2} \mathrm{O}_{2}$ levels were measured by Amplex Red fluorescence in p66shc - I- MEF and WT MEF transfected with empty FLAG expression vector or FLAG-MIA. Values are expressed relative to cells transfected with the empty FLAG vector. Expression of FLAG-MIA and endogenous p66shc is shown at bottom. (f) Lack of effect of MIA expression on active rac1 levels in WT MEF (p66shc $+1+$ ). Active GTP-rac1 was pulled down using GST-PAK1 beads, and is shown in top panel. Positive control: cell lysate treated with non-hydrolyzable $\gamma$ GTP. Negative control: cell lysate treated with GDP

MIA was cloned into 3XFLAGPCMV7.1 (Sigma) by standard methods. COS-7 and HEK 293 cells were purchased from ATCC. The Xpress-tagged cDNAs for p66shc, the $\mathrm{CH} 2$ domain, p66shcP47A/P50A, p66shcS36A, and p66shc - /- MEF have also been described previously. ${ }^{5}$ Cells were transfected using Lipofectamine 2000 (Invitrogen) as per manufacturer's recommendations.
Far-Western blot of SH3 array. An array (TransSignal SH3 Domain Array III) spotted with $\mathrm{SH} 3$ domains of several proteins, was purchased (Panomics, Redwood City, CA, USA). Recombinant (His) $\mathrm{B}_{6 \mathrm{~B}}$-tagged $\mathrm{CH} 2$ domain was induced and purified from Escherichia coli as described previously, ${ }^{29}$ and incubated with the $\mathrm{SH} 3$ domain as per manufacturer's recommendations. The far-Western blot was 
a

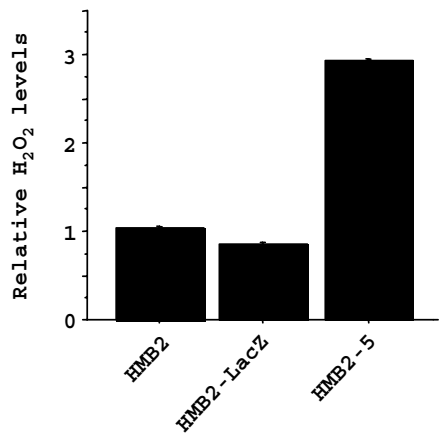

C

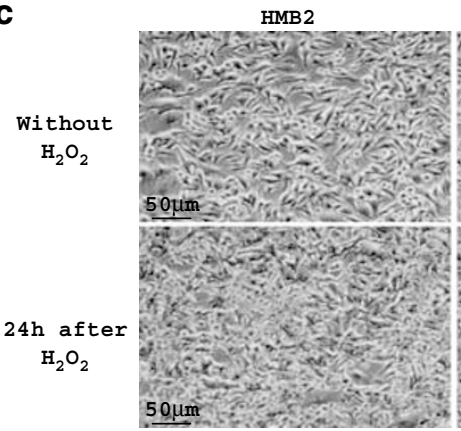

d



b

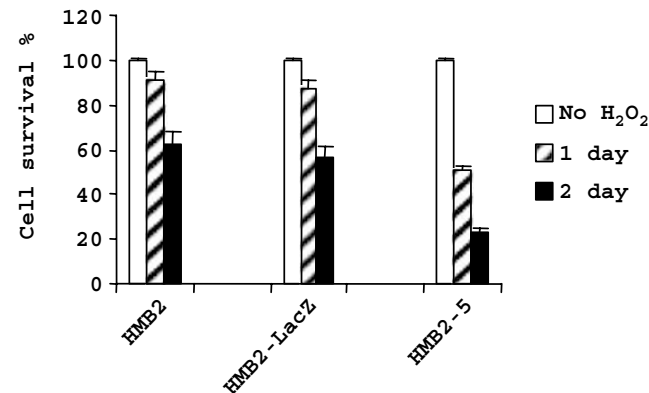

HMB2 - LacZ

HMB 2 - 5
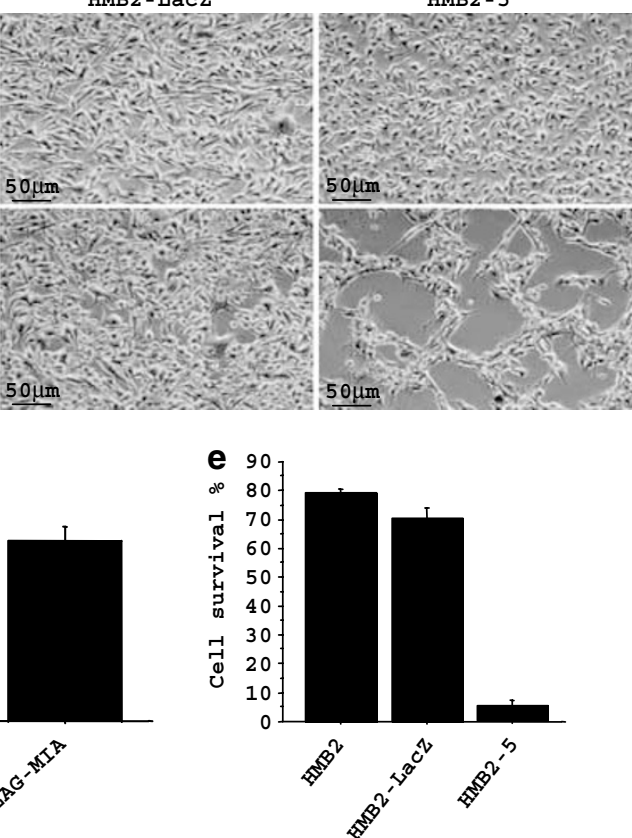

Figure 5 Endogenous MIA suppresses endogenous oxidant levels and protects melanoma cells from oxidative stress-induced death. (a) Endogenous $\mathrm{H}_{2} \mathrm{O}_{2}$ measured by Amplex Red fluorescence, in the human metastatic melanoma cell line HMB2, and its derivatives. Values are expressed relative to HMB2 cells. (b) Survival assessed by trypan blue exclusion, in $\mathrm{HMB} 2$ cells and its derivatives, 24 and $48 \mathrm{~h}$ after $0.4 \mathrm{mM} \mathrm{H}_{2} \mathrm{O}_{2}$ challenge. (c) Phase-contrast microscopic images $(\times 100)$ of $\mathrm{HMB} 2$ cells and its derivatives, $24 \mathrm{~h}$ after $0.4 \mathrm{mM} \mathrm{H}_{2} \mathrm{O}_{2}$ challenge. (d) Survival, $24 \mathrm{~h}$ after $\mathrm{H}_{2} \mathrm{O}_{2}$ challenge, assessed by trypan blue exclusion, in the MIA-deficient HMB2-5 cell line, with and without re-constitution of MIA. (e) Survival assessed by trypan blue exclusion, in HMB2 cells and its derivatives, $24 \mathrm{~h}$ after UV irradiation $\left(254 \mathrm{nM} \mathrm{UVC}\right.$ at $60 \mathrm{~J} / \mathrm{m}^{2}$ for $\left.10 \mathrm{~min} \mathrm{P}^{\mathrm{P}}\right)$

performed using an anti-His antibody (Qiagen), and the appropriate secondary antibody.

Antibodies and immunoblotting. Antibodies to X-press (Invitrogen), FLAG (Stratagene), MIA (R\&D Systems, Inc.), shc (BD Biosciences), phospho-S36p66shc (Axxora, LLC), JNK (Cell Signaling), and phospho-JNK (Cell Signaling) were purchased and used as recommended by the manufacturer. Appropriate speciesspecific secondary antibodies were used. SDS-PAGE and transfer were carried out by standard protocols. Immunoblots were developed using super Femto substrate (Pierce), and images visualized and digitally captured with a BioRad ChemiDoc apparatus.

Immunoprecipitations. Immunoprecipitations were carried out by standard methods as described previously, ${ }^{5}$ typically using $0.5-1.0 \mathrm{mg}$ of protein lysate, $2 \mu \mathrm{g}$ of antibody, and protein A-sepharose or protein G-sepharose (Amersham). Nonimmune IgG was used as a control. Immunoprecipitates were extensively washed before SDS-PAGE and immunoblotting.

Fluorescence microscopy. MIA cloned into the DsRed vector (Clonetech), and p66shc cloned into the EGFP vector (Clonetech) were co-transfected into COS-
7 cells. Red and green fluorescence images were captured on a Carl Zeiss Axiovert 200 Fluorescence microscope, and merged. Nuclei were counterstained with Hoechst 33342.

Cell death assays. Cell viability (survival) was determined using a standard Trypan Blue exclusion assay. Cell death (OD 405) was quantified using the Cell Death ELISA (Roche), and normalized for cell number (total protein).

Cell fractionation. HMB-2 cells were homogenized with a glass-Dounce homogenizer, and homogenate was separated into nuclear, mitochondrial, and ER fractions as described previously. ${ }^{30-32}$

Rac1 activity. Active GTP-bound rac1 was measured using a rac1 activity assay (UBI), as described previously. ${ }^{5}$

$\mathrm{H}_{2} \mathrm{O}_{2}$ measurements. Hydrogen peroxide $\left(\mathrm{H}_{2} \mathrm{O}_{2}\right)$ was measured in cell medium with the Amplex Red assay (Molecular Probes) as described previously. ${ }^{5}$ Endogenous $\mathrm{H}_{2} \mathrm{O}_{2}$ levels were measured by fluorescence at $595 \mathrm{~nm} 15 \mathrm{~min}$ after addition of Amplex reagent. Fluorescence intensities were normalized for cell number (total protein). 
a

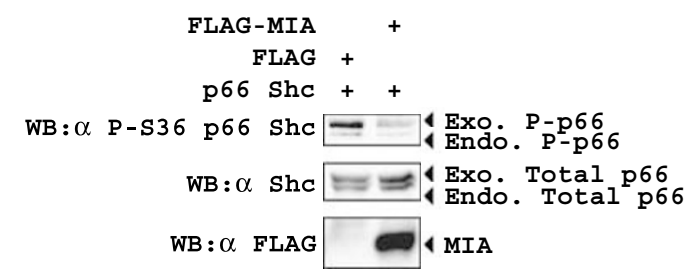

C



b

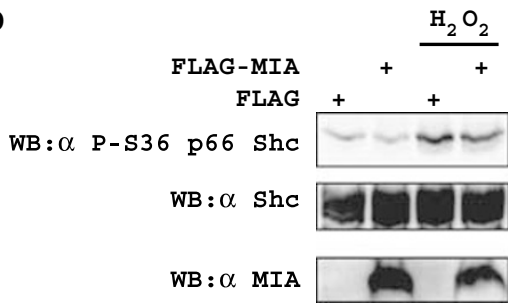

d

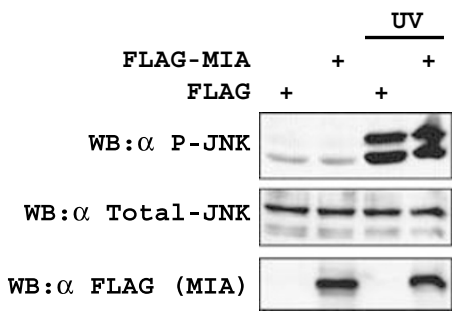

Figure 6 Ectopic MIA inhibits phosphorylation of p66Shc on Serine 36. (a) Basal phosphorylation on serine 36 (S36) of endogenous and ectopic p66shc, with and without MIA expression, in COS-7 cells (b) $\mathrm{H}_{2} \mathrm{O}_{2}$-induced phosphorylation on $\mathrm{S} 36$ of endogenous p66shc, with and without MIA expression, in COS-7 cells. Cells were challenged with $0.4 \mathrm{mM} \mathrm{H}_{2} \mathrm{O}_{2}$ for $30 \mathrm{~min}$. (c) Lack of effect of MIA on $\mathrm{H}_{2} \mathrm{O}_{2}$-induced phosphorylation of c-jun N-terminal kinase in COS-7. Cells, with and without MIA, were challenged with $0.4 \mathrm{mM} \mathrm{H}_{2} \mathrm{O}_{2}$ for $30 \mathrm{~min}$. (d) Lack of effect of MIA on UV radiation-induced phosphorylation of c-jun N-terminal kinase in COS-7. Cells, with and without MIA, were challenged with UV irradiation (UVC $254 \mathrm{nM}$ at $60 \mathrm{~J} / \mathrm{m}^{2}$ for10 $\mathrm{min}$ )

C

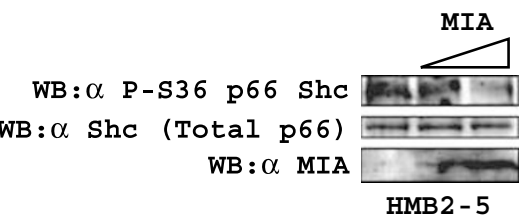

b

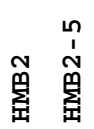

WB: $\alpha$ P-S36 p66 Shc

WB: $\alpha$ Shc (Total p66)

WB : $\alpha$ MIA

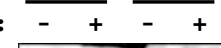

WB : $\alpha$ P-S36 p66 Shc

WB: $\alpha$ Shc (Total p66)

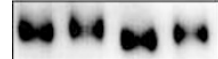

d

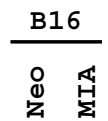

WB: $\alpha$ P-S36 p66 Shc WB: $\alpha$ Shc (Total p66)

WB : $\alpha$ MIA

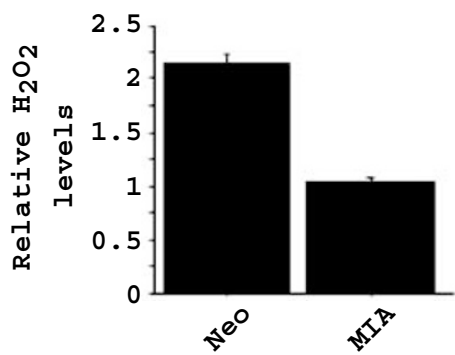

Figure 7 Endogenous MIA inhibits phosphorylation of p66Shc on Serine 36 in melanoma cells. (a) Basal phosphorylation on S36 of endogenous p66shc in the MIAexpressing HMB2 metastatic human melanoma cell line, and its MIA-deficient derivative HMB2-5. (b) UV radiation-induced phosphorylation in the MIA-expressing HMB2-LacZ and the MIA-deficient HMB2-5 human melanoma cell lines. Cells were challenged with UV irradiation (UVC $254 \mathrm{nM}$ at $60 \mathrm{~J} / \mathrm{m}^{2}$ for $10 \mathrm{~min}$ ). (c) Effect of MIA re-constitution on basal phosphorylation of S36 of endogenous p66shc in the MIA-deficient HMB2-5 cell line. (d) Effect of MIA expression on basal phosphorylation of S36 of endogenous p66shc, and basal $\mathrm{H}_{2} \mathrm{O}_{2}$ levels, in the B16 murine melanoma cell lines. MIA: MIA-expressing B16 cell line; Neo: control neomycin B16 cell line 
Acknowledgements. We thank T Finkel and S Nemoto for their generosity in providing the expression plasmids encoding p66shc, and the p66shc $-1-$ and WT MEF.

1. Migliaccio E, Giorgio M, Mele S, Pelicci G, Reboldi P, Pandolfi PP et al. The p66shc adaptor protein controls oxidative stress response and life span in mammals. Nature 1999 402: 309-313.

2. Pellegrini M, Pacini S, Baldari CT. p66SHC: the apoptotic side of Shc proteins. Apoptosis 2005; 10: 13-18.

3. Nemoto S, Finkel T. Redox regulation of forkhead proteins through a p66shc-dependent signaling pathway. Science 2002; 295: 2450-2452.

4. Trinei M, Giorgio M, Cicalese A, Barozzi S, Ventura A, Migliaccio E et al. A p53-p66Shc signalling pathway controls intracellular redox status, levels of oxidation-damaged DNA and oxidative stress-induced apoptosis. Oncogene 2002; 21: 3872-3878.

5. Khanday FA, Santhanam L, Kasuno K, Yamamori T, Naqvi A, Dericco J et al. Sosmediated activation of rac1 by p66shc. J Cell Biol 2006; 172: 817-822.

6. Blesch A, Bosserhoff AK, Apfel R, Behl C, Hessdoerfer B, Schmitt A et al. Cloning of a novel malignant melanoma-derived growth-regulatory protein, MIA. Cancer Res 1994; 54 : 5695-5701.

7. Stoll R, Renner C, Zweckstetter M, Bruggert M, Ambrosius D, Palme $S$ et al. The extracellular human melanoma inhibitory activity (MIA) protein adopts an $\mathrm{SH} 3$ domain-like fold. EMBO J 2001; 20: 340-349.

8. Bauer R, Humphries M, Fassler R, Winklmeier A, Craig SE, Bosserhoff AK. Regulation of integrin activity by MIA. J Biol Chem 2006; 281: 11669-11677.

9. Bosserhoff AK, Echtenacher B, Hein R, Buettner R. Functional role of melanoma inhibitory activity in regulating invasion and metastasis of malignant melanoma cells in vivo. Melanoma Res 2001; 11: 417-421.

10. Aung PP, Oue N, Mitani Y, Nakayama H, Yoshida K, Noguchi T et al. Systematic search for gastric cancer-specific genes based on SAGE data: melanoma inhibitory activity and matrix metalloproteinase-10 are novel prognostic factors in patients with gastric cancer Oncogene 2006; 25: 2546-2557.

11. El Fitori J, Kleeff J, Giese NA, Guweidhi A, Bosserhoff AK, Buchler MW et al. Melanoma inhibitory activity (MIA) increases the invasiveness of pancreatic cancer cells. Cancer Cell Int 2005; 5 : 3.

12. Glienke J, Schmitt AO, Pilarsky C, Hinzmann B, Weiss B, Rosenthal A et al. Differential gene expression by endothelial cells in distinct angiogenic states. Eur J Biochem 2000 267: $2820-2830$.

13. Hau $P$, Ruemmele $P$, Kunz-Schughart LA, Doerfelt A, Hirschmann $B$, Lohmeier $A$ et al. Expression levels of melanoma inhibitory activity correlate with time to progression in patients with high-grade glioma. Oncol Rep 2004; 12: 1355-1364.

14. Muller-Ladner U, Bosserhoff AK, Dreher K, Hein R, Neidhart M, Gay S et al. MIA (melanoma inhibitory activity): a potential serum marker for rheumatoid arthritis. Rheumatology (Oxford) 1999; 38: 148-154.

15. Skamrov AV, Nechaenko MA, Goryunova LE, Feoktistova ES, Khaspekov GL, Kovalevsky DA et al. Gene expression analysis to identify mRNA markers of cardiac myxoma. J Mol Cell Cardiol 2004; 37: 717-733.
16. Bosserhoff AK, Hauschild A, Hein R, Schadendorf D, Stockfleth $E$, Bogenrieder $T$ et al. Elevated MIA serum levels are of relevance for management of metastasized malignant melanomas: results of a German multicenter study. J Invest Dermatol 2000; 114: 395-396.

17. Bosserhoff AK, Kaufmann M, Kaluza B, Bartke I, Zirngibl H, Hein R et al. Melanomainhibiting activity, a novel serum marker for progression of malignant melanoma. Cancer Res 1997; 57: 3149-3153.

18. Tatzel J, Poser I, Schroeder J, Bosserhoff AK. Inhibition of melanoma inhibitory activity (MIA) expression in melanoma cells leads to molecular and phenotypic changes. Pigment Cell Res 2005; 18: 92-101.

19. Pacini S, Pellegrini M, Migliaccio E, Patrussi L, Ulivieri C, Ventura A et al. p66SHC promotes apoptosis and antagonizes mitogenic signaling in T cells. Mol Cell Biol 2004; 24: 1747-1757.

20. Le S, Connors TJ, Maroney AC. C-Jun N-terminal kinase specifically phosphorylates p66ShcA at serine 36 in response to ultraviolet irradiation. J Biol Chem 2001; 276 : $48332-48336$.

21. Kim JW, Wong CW, Goldsmith JD, Song C, Fu W, Allion MB et al. Rapid apoptosis in the pulmonary vasculature distinguishes non-metastatic from metastatic melanoma cells. Cancer Lett 2004; 213: 203-212.

22. van Groningen JJ, Bloemers HP, Swart GW. Identification of melanoma inhibitory activity and other differentially expressed messenger RNAs in human melanoma cell lines with different metastatic capacity by messenger RNA differential display. Cancer Res 1995; 55 : 6237-6243.

23. Veeramani S, Igawa T, Yuan TC, Lin FF, Lee MS, Lin JS et al. Expression of p66(Shc) protein correlates with proliferation of human prostate cancer cells. Oncogene 2005; 24: 7203-7212.

24. Bosserhoff AK, Stoll R, Sleeman JP, Bataille F, Buettner R, Holak TA. Active detachment involves inhibition of cell-matrix contacts of malignant melanoma cells by secretion of melanoma inhibitory activity. Lab Invest 2003; 83: 1583-1594.

25. Bosserhoff AK, Kondo S, Moser M, Dietz UH, Copeland NG, Gilbert DJ et al. Mouse CDRAP/MIA gene: structure, chromosomal localization, and expression in cartilage and chondrosarcoma. Dev Dyn 1997; 208: 516-525.

26. Cohen-Salmon M, Frenz D, Liu W, Verpy E, Voegeling S, Petit C. Fdp, a new fibrocytederived protein related to MIA/CD-RAP, has an in vitro effect on the early differentiation of the inner ear mesenchyme. J Biol Chem 2000; 275: 40036-40041.

27. Bosserhoff AK, Moser M, Scholmerich J, Buettner R, Hellerbrand C. Specific expression and regulation of the new melanoma inhibitory activity-related gene MIA2 in hepatocytes. J Biol Chem 2003; 278: 15225-15231.

28. Bosserhoff AK, Moser M, Buettner R. Characterization and expression pattern of the novel MIA homolog TANGO. Gene Expr Patterns 2004; 4: 473-479.

29. Khanday FA, Yamamori T, Mattagajasingh I, Zhang Z, Bugayenko A, Naqvi A et al. Rac1 leads to phosphorylation-dependent increase in stability of the p66shc adaptor protein: role in Rac1-induced oxidative stress. Mol Biol Cell 2006; 17: 122-129.

30. Gram TE. Separation of hepatic smooth and rough microsomes associated with drugmetabolizing enzymes. Methods Enzymol 1974; 31 (Pt A): 225-237.

31. Guerra FC. Rapid isolation techniques for mitochondria: technique for rat liver mitochondria. Methods Enzymol 1974; 31 (Pt A): 299-305

32. Tata JR. Isolation of nuclei from liver and other tissues. Methods Enzymol 1974; 31 (Pt A): 253-262. 